\title{
Opportunistic Scheduling with Partial Channel Information in OFDMA/FDD Systems
}

\author{
Zhong-Hai Han \\ Telecommunication R\&D Center \\ Samsung Electronics Co., LTD. \\ 416, Youngtong-Dong, Suwon 442-600, Korea \\ E-mail: zhonghai.han@samsung.com
}

\author{
Yong-Hwan Lee \\ School of Electrical Engineering and INMC \\ Seoul National University \\ Kwanak P. O. Box 34, Seoul 151-600, Korea \\ E-mail:ylee@snu.ac.kr
}

\begin{abstract}
Opportunistic scheduling (OS) is one of key techniques to increase the spectral efficiency in wireless packet-based systems by providing a multi-user diversity gain. However, when the OS is employed in orthogonal frequency division multiple access/frequency division duplex (OFDMA/FDD) systems, it may have implementation problems due to the need of a large amount of feedback information on the channel state information (CSI). To alleviate this implementation problem, we propose OS schemes that use partial CSI. The performance of the proposed OS schemes is verified by computer simulation. Simulation results show that the use of partial CSI can remarkably reduce the feedback signaling burden without significant performance degradation compared to the use of full CSI, making it quite practical to real applications.
\end{abstract}

\section{INTRODUCTION}

As the demand for data communications increases with an explosive growth of the Internet access and interactive multimedia services, broadband wireless access techniques has attracted much attention. For data services, it is well recognized that the packet-based access system have advantages over the circuit-based one, providing high transmission throughput with efficient use of radio resources. The packet scheduling is one of key technologies for efficient sharing of radio resources [1].

Most of classic studies for the packet scheduling have considered the optimization in the network-layer aspect in terms of the operation parameters such as the traffic type, quality-of services (QoS), and data rate. In packet-based wireless access systems, the use of packet scheduling makes it possible to obtain a spectral efficiency in fading channel higher than in static channel by exploiting independent fading characteristics of each mobile user (i.e., so-called multi-user diversity (MUD)) [2]. In fact, the opportunistic scheduling (OS) technique is considered as a key technology for the next generation wireless communication systems. Cdma1x EvDO system has adopted an OS scheme [2], where a proportional fairness (PF)-type scheduler allocates the user packets into the radio resource in a time-division multiplexing (TDM) manner.

When an OS scheme is employed in an orthogonal frequency division multiplexing (OFDM) system with adaptive modulation and coding (AMC), it may encounter implementation problems due to the need of a large amount of channel information. Although a number of researches were reported on adaptive bit loading and sub-carrier allocation schemes $[3,4]$, they may not be realizable due to a large amount of feedback signaling cost. To alleviate the problem due to the use of full information for the OS, we consider the use of partial channel information. We propose OS schemes based on two partial information reporting schemes, called best and fixed reporting scheme. It is shown that the OS performance with the use of partial channel information is the same as that with the use of full channel information as the number of users goes to infinity.

Following Introduction, the system model is described in Section II. The proposed scheme is described in Section III. The performance of the proposed OS scheme is verified by computer simulation in Section IV. Finally, conclusions are summarized in Section V.

\section{OPPORTUNISTIC SHEDULING IN OFDMA/FDD SYSTEM}

In this paper, we consider the transmission of packet data over an OFDMA/FDD downlink system with AMC as illustrated in Fig. 1. The packet scheduler in the base station allocates the user packet data to the radio resource based on the received channel information. We assume that the opportunistic scheduler only considers the instantaneous carrier-to-interference ratio (CIR) of each user as the channel information. Since the channel condition of each subchannel is time-varying in mobile environment, we consider the use of a dedicated signaling channel in the uplink for feedback of the CIR. The CIR is also used for AMC associated with the scheduling. We also assume the use of a dedicated signaling channel in the downlink for transmission of the scheduling and $\mathrm{AMC}$ information to the mobile.

In the OFDMA system, time and frequency division multiplexing schemes are used for efficient utilization of radio resources. The radio resources are dynamically allocated to the users by a scheduling policy based on the CIR matrix as shown in Fig. 2, where $\mathrm{V}_{i, j}$ denotes the $j$-th user's CIR of the $i$-th subchannel, $\mathrm{V}_{i, j}^{r}$ is the maximum CIR in the $i$-th row, $\mathrm{V}_{i, j}^{c}$ is

This work was in part supported by Samsung's research grant. 


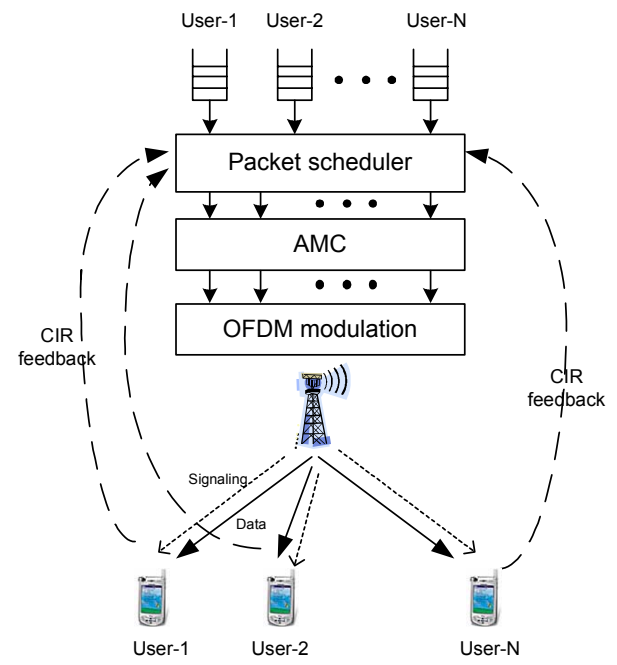

Fig. 1. OFDM system with opportunistic scheduling

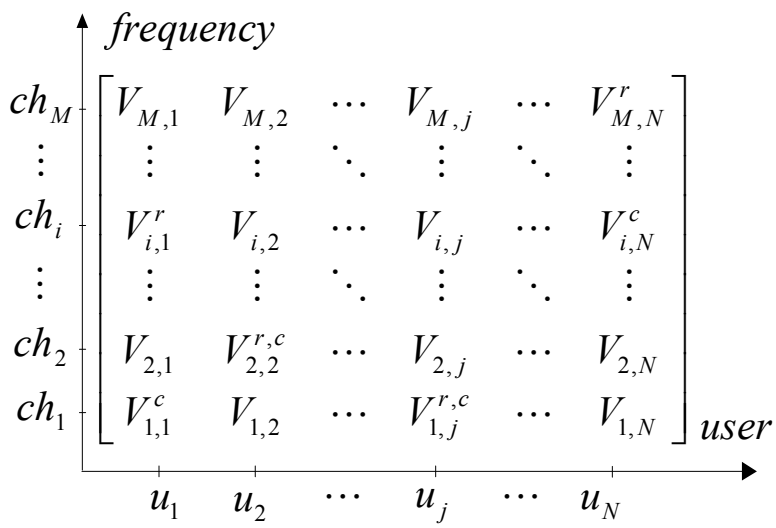

Fig. 2. The channel state information matrix

the maximum CIR in the $j$-th column, and $\mathrm{V}_{i, j}^{r, c}$ is the maximum CIR in both the $i$-th row and $j$-th column.

We consider the use of an opportunistic scheduler that selects a user in each subchannel using a relatively best (RB) policy [5], as shown in Fig. 3. The RB scheduling policy for the $i$-th subchannel can be expressed as

$$
Q_{i}\left(\mathbf{V}_{i}\right)=\arg \max _{j}\left[V_{i, j}-\bar{V}_{j}\right],
$$

where $\mathbf{V}_{i}=\left[V_{i, 1}, V_{i, 2}, \cdots, V_{i, n}\right]$ and $\bar{V}_{j}$ denotes the average CIR of the $j$-th user. The RB scheduler can significantly improve the throughput, while asymptotically providing the same fairness as the round robin scheduler [5].

When an OS scheme is employed to the OFDMA/FDD system, it requires the instantaneous CIR of all the subchannels and users, causing a large amount of feedback signaling

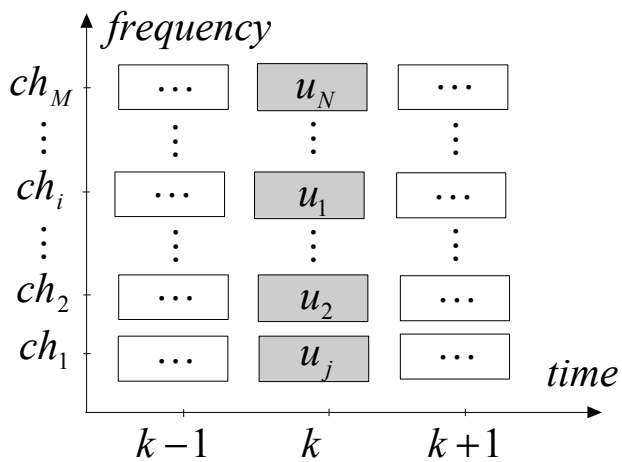

Fig. 3. An example of opportunistic scheduling with relatively best policy

burden [6]. Moreover, when the mobile speed increases, the amount of feedback information needs to be increased to keep the track of channel variation [7]. Let $N$ be the number of users, $M$ be the number of sub-channels, $T$ be the length of the time slot, and $B$ be the number of bits used for the instantaneous CIR. The total amount of feedback signaling overhead is $N M B / T$ bps. Thus, the signaling overhead linearly increases as the number of users or subchannels increases.

However, the base station may not require all the information on the instantaneous CIR. Assume that user A has a subchannel having a CIR lower than other subchannels. Then this subchannel is less likely to be assigned to user A by the scheduler. This implies that the user may not need to report the CSI of subchannels in poor condition. As the number of users increases, the scheduler can make optimum channel assignment by only considering subchannels in good condition. Thus, the user may suffice to report the channel information of subchannels in good condition, significantly reducing the feedback signaling burden.

\section{OPPORTUNISTIC SCHEDULING WITH PARTIAL CSI}

We consider a scheduling scheme that uses the CIR of $K$ subchannels instead of all $M$ subchannels, reducing the feedback signaling burden by a factor of $K / M$. We propose two types of partial information reporting schemes, called best and fixed reporting scheme. In the best reporting scheme, each user reports the CIR of $K$ subchannels having the highest instantaneous CIR. In the fixed reporting scheme, each user reports the CIR of $K$ subchannels pre-determined during the initiation or handshaking process. Unlike the best reporting scheme, it does not need to send the subchannel identification information.

When the proposed schemes are employed, there can be some subchannels without the instantaneous CIR information reported from any user. We call them empty subchannels. Since the empty subchannel has no instantaneous CIR, it cannot be applied to the opportunistic scheduling. For the 
scheduling of these empty subchannels, we use the QoS or average CIR information $\left(\bar{V}_{j}\right)$ of each user that can be known to the base station. For example, the empty channel can be assigned to a user who has the largest average CIR value or the most urgent packets to meet the QoS requirements. A simple round-robin scheduling scheme can be employed for allocation of these empty channels. The AMC level for these empty subchannels can be determined based on the average CIR value.

\section{COMPUTER SIMULATION}

The performance of the proposed scheduling scheme is verified in terms of the throughput performance by computer simulation. For the simulation, we assume that the users are uniformly distributed in a hexagonal cell, and that the OFDM signal is transmitted by 4/16/64 QAM with AMC, using 32 subchannels in $100 \mathrm{MHz}$ bandwidth. A conventional roundrobin scheduler is employed for the empty subchannel.

Fig. 4 depicts the throughput performance of the proposed scheduling schemes as a function of the number of users. For comparison, we also consider the use of full CSI for the scheduling. It can be seen that the throughput performance increases as the number of users increases due to the effect of multi-user diversity. The fixed reporting scheme is not efficient as much as the best reporting scheme, because it may not achieve the band selection gain of the user. It can also be seen that, when the number of users is small, the best reporting scheme results in noticeable performance degradation compared to the full reporting scheme since there can be many empty subchannels. However, when the number of users is large, the performance degradation is negligible because the occurrence probability of empty subchannels is very low. In fact, as the number of users goes to infinity, the OS with the use of the best reporting scheme the same throughput as that with the use of full channel information. The proof is given in Appendix.

\section{CONCLUSIONS}

The OS techniques can significantly improve the performance in a packet-based wireless communication system, but they need all the CSI of each user. In this paper, we have proposed OS schemes that use partial channel information in OFDM systems with AMC to reduce the feedback signaling burden. It has been shown that the scheduler with the use of partial channel information can provide the same performance as that with the use of full channel information as the number of users approaches to infinity. Simulation results show that the use of the best reporting scheme is quite practical to mobile environments.

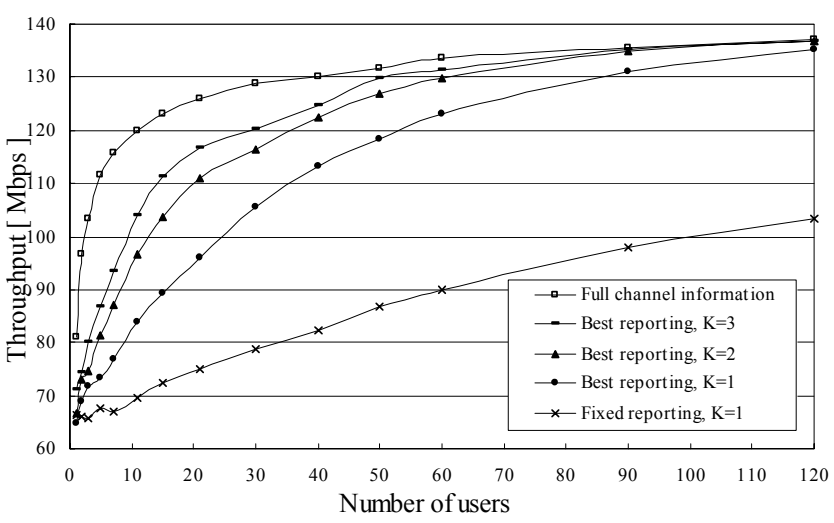

Fig. 4. The throughput performance of the proposed scheduling schemes

\section{APPENDIX}

We prove the fact that, as the number of users goes to infinity, the opportunistic scheduling scheme with the use of the best reporting scheme has the same throughput as that with the use of full channel information, by showing that the scheduling result with the use of partial CIR information is the same as that with the use of full CIR information. Let $P_{M}^{K}$ be the probability that both the schedulers come up with the same scheduling. If the scheduler with $K=1$ provides the same scheduling result as that with full CIR information, it can have the same results for $K>1$. Thus, it suffices to prove that $P_{M}^{1}$ goes to one as $N$ approaches to infinity.

Consider an $(M \times N)$ CIR matrix as shown in Fig. A1, where the $(i, j)$-th element represents the instantaneous CIR of the $j$-th user at the $i$-th subchannel. To make both the scheduling results equal, the maximum element of each row should also be the maximum one of its column since there is only one element in each column in the proposed scheme with $K=1$. Thus, $P_{M}^{1}$ can be considered as the existence probability of $M$ elements corresponding to the maximum element in both the row and column. Fig. A1 illustrates a case where $\left\{V_{i, m-i+1}^{r, c}\right\}, V_{m, 1}^{r, c}>\cdots>V_{i, m-i+1}^{r, c}>\cdots>V_{1, m}^{r, c}$, are the elements corresponding to the maximum element in both the row and column, and they are located in the diagonal position. Since there can be $M ! N ! /(N-M)$ ! possible combinations of ordering and column permutations, $P_{M}^{1}$ can be represented as

$$
P_{M}^{1}=M ! N !(N-M) ! P_{s},
$$

Where $P_{s}$ denotes the probability that the CIR matrix has a configuration in Fig. A1. Since $V_{i, m-i+1}^{r, c}$ has the maximum value among the elements enclosed by the two boxes in Fig. A1, can be obtained by 


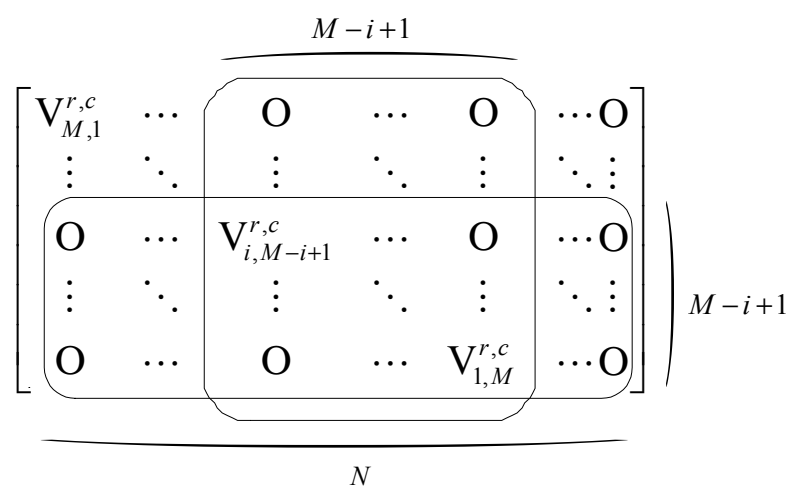

Fig. A1. An example of the CIR matrix

$$
p_{s}=\prod_{i=1}^{M} \frac{1}{N(M-i+1)+M(M-i+1)-(M-i+1)^{2}} .
$$

(A1) can be rewritten as

$$
\begin{aligned}
P_{M}^{1} & =M ! N ! /(N-M) ! \prod_{i=1}^{M} \frac{1}{(N+i-1)(M-i+1)} \\
& =\prod_{i=1}^{M} \frac{(M-i+1)(N-i+1)}{(N+i-1)(M-i+1)} \\
& =\prod_{i=0}^{M-1} \frac{N-i}{N+i} .
\end{aligned}
$$

Thus, it can be seen that

$$
\lim _{N \rightarrow \infty} P_{M}^{1}=\lim _{N \rightarrow \infty} \prod_{i=0}^{M-1} \frac{N-i}{N+i}=1
$$

[6] X. Liu, E. Chong and N. Shroff, "Optimal opportunistic scheduling in wireless networks," in Proc. of IEEE VTC'03, pp.1417- 1421, Oct. 2003.

[7] S. Catreux et. al., "Adaptive modulation and MIMO coding for broadband wireless data networks," IEEE Commun. Mag., vol. 40, pp. 108-115, June 2002.

[8] X. Liu, E. Chong and N. Shroff, "A Framework for Opportunistic Scheduling in Wireless Networks," Computer Networks, vol. 41, pp. 451-474, Mar. 2003.

[9] M. Holtzman, "Asymptotic Analysis of Proportional Fair Algorithm," in Proc. of IEEE PIMRC' 01, pp. F33-F37, Sept. 2001.

\section{REFERENCES}

[1] H. Holma and A. Toskala, WCDMA for UMTS, John Wiley \& Sons, 2000.

[2] A. Jalali, R. padovani and R. pankaj, "Date Throughput of CDMAHDR a High Efficiency-High Data Rate Personal Communication Wireless System," IEEE VTC, May 2000.

[3] J. A. C. Bingham, "Multicarrier modulation for data transmission: An idea whose time has come," IEEE Commun. Mag., pp. 5-14, May 1990.

[4] W. Rhee and J. Cioffi, "Increase in capacity of multiuser OFDM system using dynamic subchannel allocation," in Proc. of IEEE VTC'00, pp. 1085-1089, May 2000.

[5] F. Berggren and R. Jäntti, "Asymptotically fair scheduling on fading channels,” in Proc. of IEEE VTC'02 Fall, pp. 1934-1938, Sept. 2002. 